\title{
The sparse-grid combination technique applied to time-dependent advection problems
}

\author{
Boris Lastdrager*, Barry Koren, Jan Verwer \\ Center for Mathematics and Computer Science (CWI), P.O. Box 94079, 1090 GB Amsterdam, The Netherlands
}

\begin{abstract}
In the numerical technique considered in this paper, time-stepping is performed on a set of semi-coarsened space grids. At given time levels the solutions on the different space grids are combined to obtain the asymptotic convergence of a single, fine uniform grid. We present error estimates for the two-dimensional, spatially constantcoefficient model problem and discuss numerical examples. A spatially variable-coefficient problem (MolenkampCrowley test) is used to assess the practical merits of the technique. The combination technique is shown to be more efficient than the single-grid approach, yet for the Molenkamp-Crowley test, standard Richardson extrapolation is still more efficient than the combination technique. However, parallelization is expected to significantly improve the combination technique's performance. ๑ 2001 IMACS. Published by Elsevier Science B.V. All rights reserved.
\end{abstract}

Keywords: Advection problems; Sparse grids; Combination technique; Error analysis

\section{Introduction}

The long-term aim of the present work is to make significant progress in the numerical solution of large-scale transport problems: systems of partial differential equations of the advection-diffusionreaction type, used in the modeling of pollution of the atmosphere, surface water and ground water. The three-dimensional nature of these models and the necessity of modeling transport and chemical exchange between different components over long time spans, requires very efficient algorithms. For advanced three-dimensional modeling, computer capacity (computing time and memory) still is a severe limiting factor (e.g., see [8]). This limitation is felt in particular in the area of global air pollution modeling where the three-dimensional nature leads to huge numbers of grid points in each of which many calculations must be carried out. The application of sparse-grid techniques might offer a promising way out.

\footnotetext{
The current research was performed under the research contract 613-02-036 of the Netherlands Organization for Scientific Research (NWO).

* Corresponding author.

E-mail addresses: Boris.Lastdrager@CWI.nl (B. Lastdrager), Barry.Koren@CWI.nl (B. Koren), Jan.Verwer@CWI.nl (J. Verwer).
} 
Sparse-grid techniques were introduced by Zenger [10] in 1990 to reduce the number of degrees of freedom in finite-element calculations. The combination technique, as introduced in 1992 by Griebel et al. [4], can be seen as a practical implementation of the sparse-grid technique. In the combination technique, the final solution is a linear combination of solutions on semi-coarsened grids, where the coefficients of the combination are chosen such that there is a canceling in leading-order error terms. As shown by Rüde in 1993 [7], the combination technique can be placed in a broader framework of multivariate extrapolation techniques.

We show that for our two-dimensional hyperbolic problems the combination technique requires $\sim h^{-2}$ cell updates to reach an accuracy of $\mathrm{O}\left(h^{p} \log h^{-1}\right)$ while the single grid requires $\sim h^{-3}$ cell updates to solve up to an accuracy of $\mathrm{O}\left(h^{p}\right)$. Thus the combination technique is, asymptotically, more efficient than a single-grid solver. Another appealing property of the combination technique is that it is inherently parallel, i.e., it constructs the final solution from $\sim\left(\log h^{-1}\right)^{d-1}$ independent solutions ( $d$ is the dimension of the problem) which can be computed in parallel. Parallel implementations of the combination technique were shown to be effective in $[2,3]$.

Although we are ultimately interested in advection-diffusion-reaction equations, in the current work we restrict the attention to pure advection and leave the diffusion and reaction processes to future research. In a number of articles the combination technique has already been analyzed both analytically and numerically, see for instance $[1,3,4,7]$. However, in these references elliptic differential equations are considered, not hyperbolic equations like the time-dependent advection equation we are considering. In [5] the combination technique is shown to be promising for a constant coefficient advection equation. The current paper differs from [5] in that it focuses on error analysis while [5] focuses on numerical results. Furthermore, in [5] only constant coefficients are considered. Although we do not present error analysis for spatially variable coefficients, we do analyze this case numerically with the Molenkamp-Crowley test. The time-dependent coefficient case we analyze both numerically and analytically. When the combination technique is used to solve a differential equation, then a representation error and a combined discretization error are introduced. In [6] a detailed analysis is given of the representation error. In the current paper we focus on the combined discretization error.

The organization of the current paper is as follows. In Sections 2-4 we derive leading order error expressions for the error that is introduced when we solve an advection equation with spatially independent coefficients, with the combination technique. In the derivations we account for timedependent coefficients and for intermediate combinations. In Section 5 we give some estimates for the asymptotic efficiency of the combination technique relative to the single-grid approach. In Section 6 four numerical test cases are analyzed, one of these is the Molenkamp-Crowley problem. The error estimates made in the earlier sections are verified and the combination technique is compared with the single-grid technique in terms of efficiency. The conclusions are summarized in Section 7. The main conclusion is that without parallelization-although marginally-the combination technique is already more efficient than the single-grid approach for a generic advection problem, such as the Molenkamp-Crowley test. Without parallelization, the combination technique still falls behind standard Richardson extrapolation, something which has also been concluded by Rüde [7] for elliptic problems. 


\section{Discretization error}

In order to understand the combined discretization error we must first have a clear understanding of the discretization error itself. This section is devoted to the analysis of the error in the numerical solution that is due to spatial discretization. The temporal discretization errors are neglected. In the notation of functions only the relevant variables are printed, e.g., the function $f(x, y, t)$ can be referred to as $f(x, y, t), f(t), f(x, y)$ or simply as $f$, depending on context. The focus lies on the pure initial value problem for the spatially-constant coefficient, 2D advection equation

$$
c_{t}+a \partial_{x} c+b \partial_{y} c=0
$$

Eq. (1) is considered for $t=0$ up to $t=1$ and spatially discretized with finite differences on the domain $[-1,1] \times[-1,1]$. We denote the discretization of the advection operator $a \partial_{x}+b \partial_{y}$ by $a D_{x}+b D_{y}$. The corresponding spatially discretized equation reads

$$
\omega_{t}+a D_{x} \omega+b D_{y} \omega=0 .
$$

Here $\omega=\omega(t)$ still denotes a continuous function in time and space. The operators $D_{x}$ and $D_{y}$ are defined in terms of shift operators (see Section 2.2). We define the (global) discretization error $d(t)$ according to

$$
d(t) \equiv \omega(t)-c(t)
$$

We introduce the truncation error operator $E$ according to

$$
E \equiv a D_{x}+b D_{y}-a \partial_{x}-b \partial_{y} .
$$

The discretization error $d$ can be seen to satisfy

$$
d_{t}+E c_{h}+a D_{x} d+b D_{y} d=0,
$$

with general solution

$$
d(t)=\mathrm{e}^{-\int_{0}^{t}\left(a\left(t^{\prime}\right) D_{x}+b\left(t^{\prime}\right) D_{y}\right) \mathrm{d} t^{\prime}} d(0)+\left(\mathrm{e}^{-\int_{0}^{t} E\left(t^{\prime}\right) \mathrm{d} t^{\prime}}-I\right) c(t) .
$$

When $a$ and $b$ are independent of time then (5) reduces to

$$
d(t)=\mathrm{e}^{-t\left(a D_{x}+b D_{y}\right)} d(0)+\left(\mathrm{e}^{-t E}-I\right) c(t),
$$

which we expand as

$$
d(t)=\sum_{i=0}^{\infty} \frac{(-t E)^{i}}{i !} \mathrm{e}^{-t\left(a \partial_{x}+b \partial_{y}\right)} d(0)+\sum_{i=1}^{\infty} \frac{(-t E)^{i}}{i !} c(t) .
$$

\subsection{Structure of the discretization error}

In general, when the initial profile is error free it can be seen that a dimensionally split discretization of order $p$ gives rise to a discretization error given by

$$
d(t)=\sum_{i=1}^{\infty} \frac{t^{i}}{i !}\left(\sum_{j=p}^{\infty}\left(\alpha_{j} a h_{x}^{j} \partial_{x}^{j+1}+\beta_{j} b h_{y}^{j} \partial_{y}^{j+1}\right)\right)^{i} c(t),
$$


where the constants $\alpha_{j}$ and $\beta_{j}$ are the error constants in the truncation error. Eq. (7) can be rewritten in the generic form

$$
d(t)=\sum_{i=p}^{\infty}\left(h_{x}^{i} A_{i}(t)+h_{y}^{i} B_{i}(t)\right)+\sum_{j=p}^{\infty} \sum_{k=p}^{\infty} h_{x}^{j} h_{y}^{k} \gamma_{j, k}(t)
$$

showing that the discretization error consists of terms proportional to $h_{x}^{p}, h_{x}^{p+1}, \ldots$ and $h_{y}^{p}, h_{y}^{p+1}, \ldots$ and $h_{x}^{p} h_{y}^{p}, h_{x}^{p+1} h_{y}^{p}, h_{x}^{p} h_{y}^{p+1}, h_{x}^{p+1} h_{y}^{p+1}, \ldots$

\subsection{Third-order upwind discretization}

To introduce spatial discretizations we make use of the shift operators

$$
\begin{aligned}
& S_{h_{x}} f(x, y) \equiv f\left(x+h_{x}, y\right)=\sum_{i=0}^{\infty} \frac{\left(h_{x} \partial_{x}\right)^{i}}{i !} f(x, y), \\
& S_{h_{y}} f(x, y) \equiv f\left(x, y+h_{y}\right)=\sum_{i=0}^{\infty} \frac{\left(h_{y} \partial_{y}\right)^{i}}{i !} f(x, y),
\end{aligned}
$$

where we have supposed $f$ to be a $C^{\infty}$ function. We focus on the third-order upwind biased scheme which is given by

$$
D_{x}=\left\{\begin{array}{ll}
\frac{\frac{1}{6} S_{-2 h_{x}}-S_{-h_{x}}+\frac{1}{2}+\frac{1}{3} S_{h_{x}}}{h_{x}}, & a>0, \\
-\frac{\frac{1}{6} S_{2 h_{x}}-S_{h_{x}}+\frac{1}{2}+\frac{1}{3} S_{-h_{x}}}{h_{x}}, & a<0,
\end{array} \quad D_{y}= \begin{cases}\frac{\frac{1}{6} S_{-2 h_{y}}-S_{-h_{y}}+\frac{1}{2}+\frac{1}{3} S_{h_{y}}}{h_{y}}, & b>0, \\
-\frac{\frac{1}{6} S_{2 h_{y}}-S_{h_{y}}+\frac{1}{2}+\frac{1}{3} S_{-h_{y}}}{h_{y}}, & b<0 .\end{cases}\right.
$$

This yields the discretization error

$$
d(t)=\sum_{i=1}^{\infty} \frac{t^{i}}{i !}\left(\sum_{j=3}^{\infty} \frac{(-2)^{j}-3(-1)^{j}-1}{3(j+1) !}\left(\frac{a^{j+1}}{|a|^{j}} h_{x}^{j} \partial_{x}^{j+1}+\frac{b^{j+1}}{|b|^{j}} h_{y}^{j} \partial_{y}^{j+1}\right)\right)^{i} c(t),
$$

provided $d(0)=0$. Neglecting $\mathrm{O}\left(h_{x}^{4}\right)$ and $\mathrm{O}\left(h_{y}^{4}\right)$ but including $\mathrm{O}\left(h_{x}^{3} h_{y}^{3}\right)$ for later reference, Eq. (9) leads to the following leading order expression:

$$
d(t)=-\frac{t}{12}\left(|a| h_{x}^{3} \partial_{x}^{4}+|b| h_{y}^{3} \partial_{y}^{4}\right) c(t)+\frac{t^{2}}{144}|a b| h_{x}^{3} h_{y}^{3} \partial_{x}^{4} \partial_{y}^{4} c(t)+\mathrm{O}\left(h_{x}^{4}\right)+\mathrm{O}\left(h_{y}^{4}\right) .
$$

This leading-order result makes sense only when $t, a, b$ and the derivatives of $c(t)$ are moderate. The $\mathrm{O}\left(h_{x}^{3} h_{y}^{3}\right)$ term will turn out to be important since it gets amplified by the combination technique.

\subsection{Time-dependent coefficients}

To handle time-dependent coefficients we expand (5) as

$$
d(t)=\sum_{i=0}^{\infty} \frac{\left(-\int_{0}^{t} E\left(t^{\prime}\right) \mathrm{d} t^{\prime}\right)^{i}}{i !} \mathrm{e}^{-\int_{0}^{t}\left(a\left(t^{\prime}\right) \partial_{x}+b\left(t^{\prime}\right) \partial_{y}\right) \mathrm{d} t^{\prime}} d(0)+\sum_{i=1}^{\infty} \frac{\left(-\int_{0}^{t} E\left(t^{\prime}\right) \mathrm{d} t^{\prime}\right)^{i}}{i !} c(t) .
$$


For $d(0)=0$, the time-dependent equivalent to (10) then reads

$$
\begin{aligned}
d(t)= & -\frac{1}{12}\left(\int_{0}^{t}\left|a\left(t^{\prime}\right)\right| \mathrm{d} t^{\prime} h_{x}^{3} \partial_{x}^{4}+\int_{0}^{t}\left|b\left(t^{\prime}\right)\right| \mathrm{d} t^{\prime} h_{y}^{3} \partial_{y}^{4}\right) c(t) \\
& +\frac{1}{144}\left(\int_{0}^{t}\left|a\left(t^{\prime}\right)\right| \mathrm{d} t^{\prime}\right)\left(\int_{0}^{t}\left|b\left(t^{\prime}\right)\right| \mathrm{d} t^{\prime}\right) h_{x}^{3} h_{y}^{3} \partial_{x}^{4} \partial_{y}^{4} c(t)+\mathrm{O}\left(h_{x}^{4}\right)+\mathrm{O}\left(h_{y}^{4}\right) .
\end{aligned}
$$

\section{Combination technique}

The two-dimensional combination technique is based on a grid of grids as shown in Fig. 1. Grids within the grid of grids are denoted by $\Omega^{l, m}$ where upper indices label the level of refinement relative to the root grid $\Omega^{0,0}$. The mesh widths in $x$ - and $y$-direction of $\Omega^{l, m}$ are $h_{x}=2^{-l} H$ and $h_{y}=2^{-m} H$, where $H$ is the mesh width of the uniform root grid $\Omega^{0,0}$. We denote the mesh width of the finest grid $\Omega^{N, N}$ by $h$. Note that $h_{x}$ and $h_{y}$ are dependent on the position $(l, m)$ in the grid of grids while $h$ is not.

In the time-dependent combination technique a given initial profile $c(x, y, 0)$ is restricted, by injection, onto the grids $\Omega^{N, 0}, \Omega^{N-1,1}, \ldots, \Omega^{0, N}$ and onto $\Omega^{N-1,0}, \Omega^{N-2,1}, \ldots, \Omega^{0, N-1}$, see Fig. 1 . The resulting coarse representations are then all evolved in time (exact time integration is assumed in the current paper). Then, at a chosen point in time, the coarse approximations are prolongated with $q$ th order interpolation onto the finest grid $\Omega^{N, N}$, where they are combined according to (13) to obtain a more accurate solution. The notation is summarized in Fig. 1.

We use the symbol to denote the grid functions that are constructed with the combination technique. Considering the exact solution $c$, the combination technique, as introduced in [4], constructs a grid

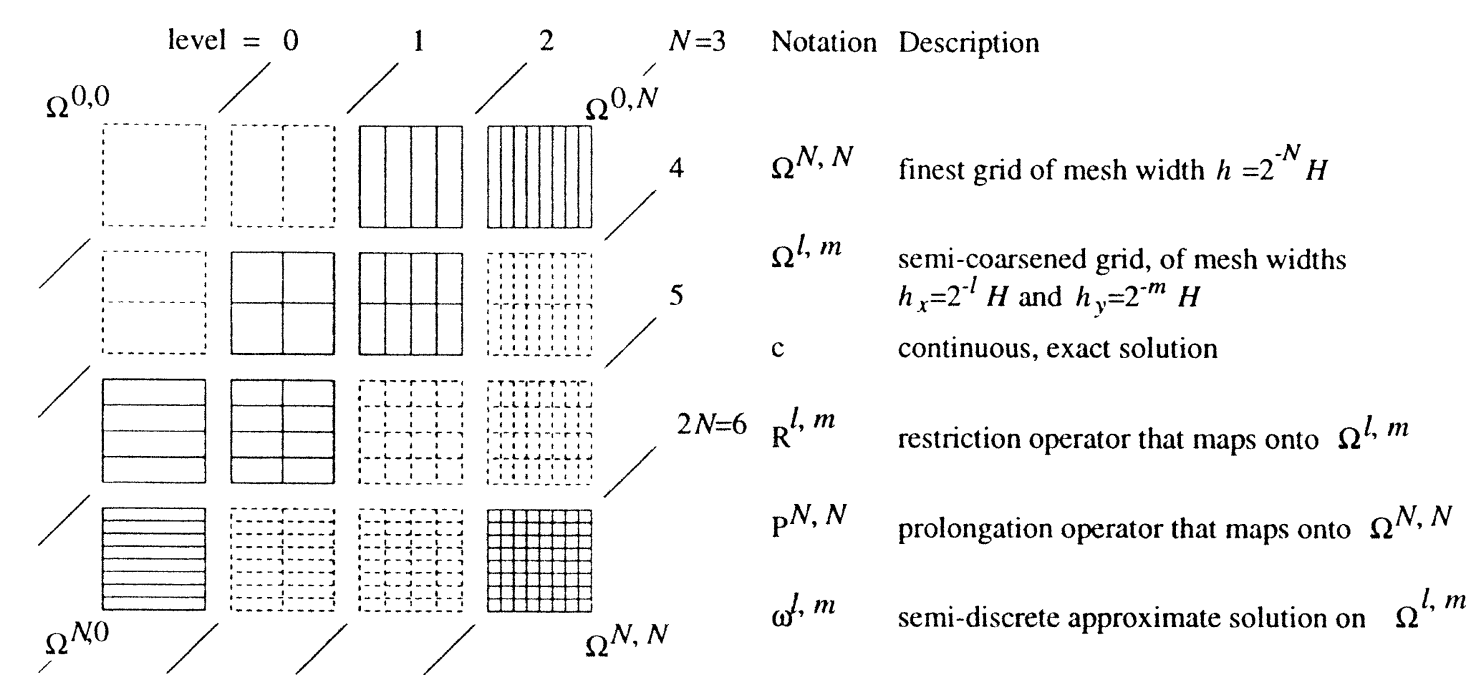

Fig. 1. Grid of grids. 
function $\widehat{c}^{N, N}$ on the finest grid $\Omega^{N, N}$ in the following manner,

$$
\widehat{c}^{N, N} \equiv \sum_{l+m=N} P^{N, N} R^{l, m} c-\sum_{l+m=N-1} P^{N, N} R^{l, m} c .
$$

The corresponding so-called representation error $r^{N, N}$ is

$$
r^{N, N} \equiv \widehat{c}^{N, N}-R^{N, N} c \text {. }
$$

Likewise, considering the semi-discrete solutions $\omega^{l, m}$, the combination technique constructs an approximate solution $\widehat{\omega}^{N, N}$ on the finest grid $\Omega^{N, N}$ from the coarse-grid approximate solutions according to

$$
\widehat{\omega}^{N, N}=\sum_{l+m=N} P^{N, N} \omega^{l, m}-\sum_{l+m=N-1} P^{N, N} \omega^{l, m} .
$$

Let $d^{l, m}$ denote the discretization error on grid $\Omega^{l, m}$, i.e.,

$$
d^{l, m} \equiv \omega^{l, m}-R^{l, m} c .
$$

The total error $e^{N, N}=\widehat{\omega}^{N, N}-R^{N, N} c$ present in $\widehat{\omega}^{N, N}$ is written as

$$
e^{N, N}=r^{N, N}+\widehat{d}^{N, N},
$$

where the combined discretization error $\widehat{d}^{N, N}=\widehat{\omega}^{N, N}-\widehat{c}^{N, N}$ is given by

$$
\widehat{d}^{N, N}=\sum_{l+m=N} P^{N, N} d^{l, m}-\sum_{l+m=N-1} P^{N, N} d^{l, m} .
$$

In [6] a detailed analysis is given of the representation error $r^{N, N}$. In the current paper we focus on the combined discretization error $\widehat{d}^{N, N}$. In Section 6 on numerical results it will become apparent that the representation error $r^{N, N}$ is negligible compared to the combined discretization error $\widehat{d}^{N, N}$.

\section{Combined discretization error}

\subsection{Effect of the combination technique on a single error term}

Inspection of (7) shows that the discretization error $d^{l, m}$ can be expanded as

$$
d^{l, m}(t)=\sum_{i=0}^{\infty} \sum_{j=0}^{\infty} h_{x}^{i} h_{y}^{j} R^{l, m} \theta_{i, j}(t) c(x, y, t),
$$

where the powers of $t$ and the spatial differential operators are hidden in $\theta_{i, j}(t)$. Eq. (16) allows us to concentrate on powers of $h_{x}$ and $h_{y}$. Since $h_{x}=2^{-l} H$ and $h_{y}=2^{-m} H$ we can rewrite (16) as

$$
d^{l, m}(t)=\sum_{i=0}^{\infty} \sum_{j=0}^{\infty} H^{i+j} \varepsilon_{i, j}^{l, m}(t),
$$

where

$$
\varepsilon_{i, j}^{l, m}(t) \equiv 2^{-i l-j m} R^{l, m} \theta_{i j}(t) c(x, y, t) .
$$


Insertion of (17) into the expression for the combined discretization error (15) yields

$$
\widehat{d}^{N, N}=\sum_{i j} H^{i+j} \widehat{\varepsilon}_{i, j}^{N, N},
$$

where

$$
\widehat{\varepsilon}_{i, j}^{N, N} \equiv \sum_{l+m=N} P^{N, N} \varepsilon_{i, j}^{l, m}-\sum_{l+m=N-1} P^{N, N} \varepsilon_{i, j}^{l, m} .
$$

We now focus on the contribution that a single error term $\varepsilon_{i, j}^{l, m}$ makes to the combined discretization error, i.e., we analyze $\widehat{\varepsilon}_{i, j}^{N, N}$ The error terms $\varepsilon_{i, j}^{l, m}$ are prolongated onto the finest grid $\Omega^{N, N}$ with interpolation of order $q$, yielding interpolation errors $\zeta_{i, j}^{N, N, l, m}$ and grid functions $\xi_{i, j}^{N, N, l, m}$ that are free of interpolation errors, i.e.,

$$
P^{N, N} \varepsilon_{i, j}^{l, m}=\xi_{i, j}^{N, N, l, m}+\zeta_{i, j}^{N, N, l, m} .
$$

The latter two superscripts in $\zeta_{i, j}^{N, N, l, m}$ and $\xi_{i, j}^{N, N, l, m}$ denote from which grid these grid functions originate. For $\widehat{\varepsilon}_{i, j}^{N, N}$ this leads to the splitting

$$
\widehat{\varepsilon}_{i, j}^{N, N}=\widehat{\xi}_{i, j}^{N, N}+\widehat{\zeta}_{i, j}^{N, N}
$$

\subsubsection{Error without interpolation effects}

According to (18) we have

$$
\xi_{i, j}^{N, N, l, m} \equiv 2^{-i l-j m} R^{N, N} \theta_{i, j} c,
$$

hence

$$
\widehat{\xi}_{i, j}^{N, N}=\left(\sum_{l+m=N}-\sum_{l+m=N-1}\right) 2^{-i l-j m} R^{N, N} \theta_{i, j} c,
$$

which is equivalent to

$$
\begin{aligned}
\widehat{\xi}_{i, j}^{N, N} & =\left(\sum_{l=0}^{N} 2^{-i l-j(N-l)}-\sum_{l=0}^{N-1} 2^{-i l-j(N-1-l)}\right) R^{N, N} \theta_{i, j} c \\
& =\left(2^{-i N}+2^{-j N}\left[1-2^{j}\right] \sum_{l=0}^{N-1} 2^{l(j-i)}\right) R^{N, N} \theta_{i, j} c .
\end{aligned}
$$

For $i=j$ this yields

$$
\widehat{\xi}_{i, j}^{N, N}=\left(2^{-i N}+2^{-i N}\left[1-2^{i}\right] N\right) R^{N, N} \theta_{i, i} c
$$

while for $i \neq j$

$$
\widehat{\xi}_{i, j}^{N, N}=\left(\frac{1}{2^{j}-2^{i}}\left[2^{-j N}\left(2^{i+j}-2^{i}\right)+2^{-i N}\left(2^{j}-2^{i+j}\right)\right]\right) R^{N, N} \theta_{i, j} c .
$$

Eqs. (20) and (21) lead to the following order estimates:

$$
\widehat{\xi}_{i, j}^{N, N}= \begin{cases}\mathrm{O}\left(2^{-j N}\right) & \text { if } i=0, j \neq 0 \\ \mathrm{O}\left(2^{-i N}\right) & \text { if } j=0, i \neq 0 \\ \mathrm{O}\left(N 2^{-i N}\right) & \text { if } i=j \neq 0 \\ \mathrm{O}\left(2^{-\min (i j) N}\right) & \text { if } i \neq j, i \neq 0, j \neq 0\end{cases}
$$




\subsubsection{Additional error due to interpolation}

In leading order the interpolation error is given by

$$
\zeta_{i, j}^{N, N, l, m}=\left(\lambda_{l} h_{x}^{q} \partial_{x}^{q}+\lambda_{m} h_{y}^{q} \partial_{y}^{q}\right) \xi_{i, j}^{N, N, l, m},
$$

or equivalently,

$$
\zeta_{i, j}^{N, N, l, m}=H^{q} R^{N, N}\left(2^{-(q+i) l-j m} \lambda_{l} \partial_{x}^{q}+2^{-(q+j) m-i l} \lambda_{m} \partial_{y}^{q}\right) \theta_{i, j} c,
$$

where the $\lambda_{l}$ and $\lambda_{m}$ are coefficients dependent on $l$ and $m$, respectively, and on the choice of interpolation. For the combined interpolation error $\widehat{\zeta}_{i, j}^{N, N}$ we have

$$
\begin{aligned}
\widehat{\zeta}_{i, j}^{N, N}= & H^{q} R^{N, N}\left(\sum_{l+m=N}-\sum_{l+m=N-1}\right) 2^{-(q+i) l-j m} \lambda_{l} \partial_{x}^{q} \theta_{i, j} c \\
& +H^{q} R^{N, N}\left(\sum_{l+m=N}-\sum_{l+m=N-1}\right) 2^{-(q+j) m-i l} \lambda_{m} \partial_{y}^{q} \theta_{i, j} c .
\end{aligned}
$$

For the first term,

$$
\left(\sum_{l+m=N}-\sum_{l+m=N-1}\right) 2^{-(q+i) l-j m} \lambda_{l} \partial_{x}^{q} \theta_{i, j} c,
$$

we obtain

$$
\left(2^{-(q+i) N} \lambda_{N}+\sum_{l=0}^{N-1}\left(2^{-(q+i) l-j(N-l)}-2^{-(q+i) l-j(N-1-l)}\right) \lambda_{l}\right) \partial_{x}^{q} \theta_{i, j} c,
$$

which, in absolute value, is bounded from above by

$$
|\lambda|_{\max }\left|\left(2^{-(q+i) N}+\sum_{l=0}^{N-1}\left(2^{-(q+i) l-j(N-l)}-2^{-(q+i) l-j(N-1-l)}\right)\right) \partial_{x}^{q} \theta_{i, j} c\right| .
$$

Likewise, the second term,

$$
\left(\sum_{l+m=N}-\sum_{l+m=N-1}\right) 2^{-(q+j) m-i l} \lambda_{m} \partial_{x}^{q} \theta_{i, j} c,
$$

is in absolute value bounded from above by

$$
|\lambda|_{\max }\left|\left(2^{-(q+j) N}+\sum_{m=0}^{N-1}\left(2^{-(q+j) m-i(N-m)}-2^{-(q+j) m-i(N-1-m)}\right)\right) \partial_{y}^{q} \theta_{i, j} c\right| .
$$

Together these bounds lead to the following order estimates, in the same way as the estimates in the previous section were obtained:

$$
\widehat{\zeta}_{i, j}^{N, N}= \begin{cases}\mathrm{O}\left(H^{q} 2^{-q N}\right) & \text { if } i=0 \text { or } j=0, \\ \mathrm{O}\left(H^{q} N 2^{-j N}\right) & \text { if } q+i=j, \\ \mathrm{O}\left(H^{q} N 2^{-i N}\right) & \text { if } q+j=i, \\ \mathrm{O}\left(H^{q} 2^{-\min (i, j) N}\right) & \text { if } 0 \neq j \neq q+i \text { and } 0 \neq i \neq q+j .\end{cases}
$$




\subsection{Leading-order results}

By combining the order estimates (22) for a single error term and Eqs. (20) and (21) with the structure of a dimensionally split discretization error (8), we see that in the discretization error the following terms are of particular interest:

$$
d=t\left(\alpha_{p} a h_{x}^{p} \partial_{x}^{p+1}+\beta_{p} b h_{y}^{p} \partial_{y}^{p+1}\right) c+t^{2} \alpha_{p} \beta_{p} a b h_{x}^{p} h_{y}^{p} \partial_{x}^{p+1} \partial_{y}^{p+1} c+\mathrm{O}\left(h_{x}^{p+1}\right)+\mathrm{O}\left(h_{y}^{p+1}\right),
$$

where we have omitted the upper indices $N, N$. To obtain the corresponding expression for the combined discretization error we use (20) and (21). The effect of the first and second terms in (24) is given by (21) with $i=p, j=0$ and $i=0, j=p$, respectively. The effect of the third term in (24) is given by (20) with $i=j=p$. Working this out leads to the following leading-order expression for the combined discretization error

$$
\begin{aligned}
\widehat{d}= & t\left(\alpha_{p} a h^{p} \partial_{x}^{p+1}+\beta_{p} b h^{p} \partial_{y}^{p+1}\right) c+t^{2} \alpha_{p} \beta_{p} a b H^{p} h^{p}\left(1+\left(1-2^{p}\right) \log _{2} \frac{H}{h}\right) \partial_{x}^{p+1} \partial_{y}^{p+1} c \\
& +\mathrm{O}\left(h^{p+1} \log _{2} \frac{1}{h}\right) .
\end{aligned}
$$

More specifically, for the third-order upwind scheme,

$$
\widehat{d}=-\frac{t h^{3}}{12}\left(|a| \partial_{x}^{4}+|b| \partial_{y}^{4}\right) c+\frac{t^{2}}{144}|a b| H^{3} h^{3}\left(1-7 \log _{2} \frac{H}{h}\right) \partial_{x}^{4} \partial_{y}^{4} c+\mathrm{O}\left(h^{4} \log _{2} \frac{1}{h}\right) .
$$

\subsection{Mapping of error terms}

We illustrate the effect of a single term of the discretization error on the error that is observed on the finest grid after applying the combination technique. We view the combination technique as a mapping that maps terms from the discretization error onto a leading-order error term on the finest grid. We assume that the order of the prolongation $q$ is greater than the order of the discretization $p$. The order estimate (22) shows that, for $i \neq j, i \neq 0, j \neq 0$, we have a mapping according to Table 1 . While the discretization error's leading-order terms, proportional to $h_{x}^{p}$ and $h_{y}^{p}$ yield error terms of $\mathrm{O}\left(h^{p}\right)$, the crossderivative term proportional to $h_{x}^{p} h_{y}^{p}$ surpasses these and yields the new formal leading-order error term proportional to $h^{p} \log h^{-1}$.

Table 1

Mapping of error terms from the semi-coarsened grids to the finest grid

\begin{tabular}{cl}
\hline Error term on $\left\{\Omega^{l, m}\right\}$ & Effect on $\Omega^{N, N}$ \\
\hline$h_{x}^{i}$ or $h_{y}^{i}$ & $\mathrm{O}\left(h^{i}\right)$ \\
$h_{x}^{i} h_{y}^{j}$ & $\mathrm{O}\left(h^{\min (i, j)}\right)$ \\
$h_{x}^{i} h_{y}^{i}$ & $\mathrm{O}\left(h^{i} \log h^{-1}\right)$ \\
\hline
\end{tabular}




\subsection{Additional error due to interpolation}

From the order estimates (23) we find that:

- if $q \neq p$ then the contribution of the interpolation error is

$$
\mathrm{O}\left(H^{p} h^{q}\right)
$$

- if $q=p$ then the contribution of the interpolation error is

$$
\mathrm{O}\left(H^{p} h^{p} \log \frac{H}{h}\right) \text {. }
$$

According to (27) the interpolation leaves the leading-order result (25) unaffected, provided the order of interpolation $q$ is greater than the order of discretization $p$. When $q=p$, according to (28), the effect of the interpolation is of the same order as the second term in the leading-order result (25). For $q<p$ the interpolation error is in fact larger than the leading-order result (25) itself. Thus choosing $q<p$ is not sensible since it leads to an order reduction in the error. Choosing $q=p$ is acceptable when the parameters of the combination technique are such that the second term in (25) is dominated by the first term. When this is not the case, $q$ must be chosen larger than $p$.

\subsection{Intermediate combinations}

When the combination technique is used in conjunction with a time-stepping technique, as we do, then we can choose to make intermediate combinations. With intermediate combinations the algorithm is as follows:

1. The initial solution is restricted to the semi-coarsened grids.

2. A number of time integration steps is performed on the semi-coarsened solutions.

3. The semi-coarsened solutions are prolongated onto and combined on the finest grid $\Omega^{N, N}$.

4. The combined solution is projected back onto the semi-coarsened grids.

5. Steps 2-4 are repeated until the time integration is completed (in the last loop, step 4 is then omitted).

We will now analyze the influence of intermediate combinations on the error, specifically we consider $M-1$ intermediate combinations made at times $t / M, 2 t / M, \ldots,(M-1) t / M$. For a single semicoarsened grid $\Omega^{l, m}$ onto which an intermediate solution was restricted at $t / M$, we have, according to $(6)$,

$$
d^{l, m}\left(\frac{2 t}{M}\right)=\sum_{j=0}^{\infty} \frac{(-(t / M) E)^{j}}{j !} \mathrm{e}^{-(t / M)\left(a \partial_{x}+b \partial_{y}\right)} R^{l, m} \widehat{d}^{N, N}\left(\frac{t}{M}\right)+\sum_{i=1}^{\infty} \frac{(-(t / M) E)^{i}}{i !} R^{l, m} c\left(\frac{2 t}{M}\right) .
$$

Due to the leading order result (25) we have

$$
\begin{aligned}
\mathrm{e}^{-(t / M)\left(a \partial_{x}+b \partial_{y}\right)} R^{l, m} \widehat{d}^{N, N}\left(\frac{t}{M}\right) \\
=\frac{t}{M}\left(\alpha_{p} a h^{p} \partial_{x}^{p+1}+\beta_{p} b h^{p} \partial_{y}^{p+1}\right) R^{l, m} c\left(\frac{2 t}{M}\right) \\
\quad+\frac{t^{2}}{M^{2}} \alpha_{p} \beta_{p} a b H^{p} h^{p}\left(1+\left(1-2^{p}\right) \log _{2} \frac{H}{h}\right) \partial_{x}^{p+1} \partial_{y}^{p+1} R^{l, m} c\left(\frac{2 t}{M}\right)+\mathrm{O}\left(h^{p+1} \log _{2} \frac{1}{h}\right) .
\end{aligned}
$$


Here we have used $\mathrm{e}^{-(t / M)\left(a \partial_{x}+b \partial_{y}\right)} c(t / M)=c(2 t / M)$. In the first summation in (29), terms with $j>0$ will only contribute in higher order because $E$ is a power expansion in mesh widths $h_{x}$ and $h_{y}$. Hence we can neglect the $j>0$ terms in (29) for a leading-order result, yielding

$$
\begin{aligned}
d^{l, m}\left(\frac{2 t}{M}\right)= & \frac{t}{M}\left(\alpha_{p} a h^{p} \partial_{x}^{p+1}+\beta_{p} b h^{p} \partial_{y}^{p+1}\right) R^{l, m} c\left(\frac{2 t}{M}\right) \\
& +\frac{t^{2}}{M^{2}} \alpha_{p} \beta_{p} a b H^{p} h^{p}\left(1+\left(1-2^{p}\right) \log _{2} \frac{H}{h}\right) \partial_{x}^{p+1} \partial_{y}^{p+1} R^{l, m} c\left(\frac{2 t}{M}\right) \\
& +\mathrm{O}\left(h^{p+1} \log _{2} \frac{1}{h}\right)+\sum_{i=1}^{\infty} \frac{(-(t / M) E)^{i}}{i !} R^{l, m} c\left(\frac{2 t}{M}\right) \\
& +\mathrm{O}\left(\left(h_{x}^{p}+h_{y}^{p}+h_{x}^{p} h_{y}^{p}\right)\left(h^{p}+h^{p} \log _{2} \frac{1}{h}\right)\right) .
\end{aligned}
$$

The above expression immediately leads to the leading-order result for the combined discretization error $\widehat{d}^{N, N}(2 t / M)$ taking into account an intermediate combination at $t / M$. The first two terms and the $\mathrm{O}\left(h^{p+1} \log _{2}(1 / h)\right)$ term carry over into $\widehat{d}^{N, N}(2 t / M)$ without alterations since we neglect representation errors. The summation yields the two terms in (25) as was argued in Sections 4.1 and 4.2. The last O-term translates according to the rules stated in Section 4.1. Thus, (30) yields the following for the combined discretization error $\widehat{d}^{N, N}(2 t / M)$ taking into account an intermediate combination at $t / M$ :

$$
\begin{aligned}
\widehat{d}^{N, N}\left(\frac{2 t}{M}\right)=2[ & \frac{t}{M}\left(\alpha_{p} a h^{p} \partial_{x}^{p+1}+\beta_{p} b h^{p} \partial_{y}^{p+1}\right) R^{N, N} c\left(\frac{2 t}{M}\right) \\
& \left.+\frac{t^{2}}{M^{2}} \alpha_{p} \beta_{p} a b H^{p} h^{p}\left(1+\left(1-2^{p}\right) \log _{2} \frac{H}{h}\right) \partial_{x}^{p+1} \partial_{y}^{p+1} R^{N, N} c\left(\frac{2 t}{M}\right)\right] \\
& +\mathrm{O}\left(h^{p+1} \log _{2} \frac{1}{h}\right)+\mathrm{O}\left(\left(h^{p}+h^{p}+h^{p} \log _{2} \frac{1}{h}\right)\left(h^{p}+h^{p} \log _{2} \frac{1}{h}\right)\right) .
\end{aligned}
$$

By induction this leads to the following result for the combined discretization error at $t$, taking into account intermediate combinations at $t / M, 2 t / M, \ldots,(M-1) t / M$,

$$
\begin{aligned}
\widehat{d}^{N . N}(t)= & t\left(\alpha_{p} a h^{p} \partial_{x}^{p+1}+\beta_{p} b h^{p} \partial_{y}^{p+1}\right) R^{N, N} c(t) \\
& +\frac{1}{M} t^{2} \alpha_{p} \beta_{p} a b H^{p} h^{p}\left(1+\left(1-2^{p}\right) \log _{2} \frac{H}{h}\right) \partial_{x}^{p+1} \partial_{y}^{p+1} R^{N, N} c(t) \\
& +\mathrm{O}\left(h^{p+1} \log _{2} \frac{1}{h}\right)
\end{aligned}
$$

i.e., the term proportional to $h^{p} \log h^{-1}$ is attenuated by a factor $1 / M$. For the third-order upwind discretization equation (32) yields

$$
\widehat{d}=-\frac{t h^{3}}{12}\left(|a| \partial_{x}^{4}+|b| \partial_{y}^{4}\right) c+\frac{t^{2}}{144 M}|a b| H^{3} h^{3}\left(1-7 \log _{2} \frac{H}{h}\right) \partial_{x}^{4} \partial_{y}^{4} c+\mathrm{O}\left(h^{4} \log _{2} \frac{1}{h}\right) .
$$

\subsection{Qualitative behavior of the error}

Provided the effects of interpolation can be neglected the error in the combined solution is given by (32). The competition between the two terms in (32) is determined by the time up to which we 
integrate, the number of combinations $M$, the coefficients $a$ and $b$, the root mesh width $H$, the number of grids (through $\log _{2}(H / h)$ ), the order of discretization $p$ (through $\alpha_{p}, \beta_{p}$ and $2^{p}$ ) and by the derivatives of the exact solution. Given this multitude of dependencies it seems likely that in general both terms can be important in describing the error.

When $a \approx b$ (i.e., advection diagonal to the grid) or when the exact solution has a large cross derivative $\partial_{x}^{p+1} \partial_{y}^{p+1} c$ compared to the derivatives $\partial_{x}^{p+1} c$ and $\partial_{y}^{p+1} c$, then the second term in (32) gains importance. Since this term represents the additional error due to using the combination technique, rather than a single grid, we see that the combination technique is less well suited to problems with $a \approx b$ or with large cross derivatives. Both are features of a problem that is not grid-aligned, i.e., the combination technique works better for grid-aligned problems.

We mention two mechanisms that will attenuate the second term in (32). First, the semi-coarsened grids used in the combination technique need to be sufficiently fine to describe the solution. This requires $H$ to be small and thus attenuates the second term in (32), which has $H^{p}$ as a prefactor. Second, it is a practical observation that a number of intermediate combinations $(M-1)$ is needed to successfully apply the combination technique, causing a further reduction of the second term by a factor $1 / M$.

\subsection{Time-dependent coefficients}

Up to now the results in the current section are valid for coefficients that are independent of time. We now state the leading-order results for time-dependent coefficients. The statements about the interpolation error still hold. The leading-order expression for the combined discretization error becomes

$$
\begin{aligned}
\widehat{d}= & \left(\int_{0}^{t} \alpha_{p}\left(t^{\prime}\right) a\left(t^{\prime}\right) \mathrm{d} t^{\prime}\right) h^{p} \partial_{x}^{p+1} c+\left(\int_{0}^{t} \beta_{p}\left(t^{\prime}\right) b\left(t^{\prime}\right) \mathrm{d} t^{\prime}\right) h^{p} \partial_{y}^{p+1} c \\
& +\left(\int_{0}^{t} \alpha_{p}\left(t^{\prime}\right) a\left(t^{\prime}\right) \mathrm{d} t^{\prime}\right)\left(\int_{0}^{t} \beta_{p}\left(t^{\prime}\right) b\left(t^{\prime}\right) \mathrm{d} t^{\prime}\right) H^{p} h^{p}\left(1+\left(1-2^{p}\right) \log _{2} \frac{H}{h}\right) \partial_{x}^{p+1} \partial_{y}^{p+1} c \\
& +\mathrm{O}\left(h^{p+1} \log _{2} \frac{1}{h}\right) .
\end{aligned}
$$

For third-order upwind discretization this yields

$$
\begin{aligned}
\widehat{d}= & -\frac{h^{3}}{12}\left(\int_{0}^{t}\left|\alpha\left(t^{\prime}\right)\right| \mathrm{d} t^{\prime} \partial_{x}^{4}+\int_{0}^{t}\left|b\left(t^{\prime}\right)\right| \mathrm{d} t^{\prime} \partial_{y}^{4}\right) c \\
& +\frac{H^{p} h^{p}}{144}\left(1+\left(1-2^{p}\right) \log _{2} \frac{H}{h}\right)\left(\int_{0}^{t}\left|a\left(t^{\prime}\right)\right| \mathrm{d} t^{\prime}\right)\left(\int_{0}^{t}\left|b\left(t^{\prime}\right)\right| \mathrm{d} t^{\prime}\right) \partial_{x}^{4} \partial_{y}^{4} c+\mathrm{O}\left(h^{4} \log _{2} \frac{1}{h}\right) .
\end{aligned}
$$

When $M-1$ intermediate combinations are made, the combined discretization error is given by

$$
\widehat{d}=\left(\int_{0}^{t} \alpha_{p}\left(t^{\prime}\right) a\left(t^{\prime}\right) \mathrm{d} t^{\prime}\right) h^{p} \partial_{x}^{p+1} c+\left(\int_{0}^{t} \beta_{p}\left(t^{\prime}\right) b\left(t^{\prime}\right) \mathrm{d} t^{\prime}\right) h^{p} \partial_{y}^{p+1} c
$$




$$
\begin{aligned}
& +\sum_{n=0}^{M-1}\left(\int_{n t / M}^{(n+1) t / M} \alpha_{p}\left(t^{\prime}\right) a\left(t^{\prime}\right) \mathrm{d} t^{\prime}\right)\left(\int_{n t / M}^{(n+1) t / M} \beta_{p}\left(t^{\prime}\right) b\left(t^{\prime}\right) \mathrm{d} t^{\prime}\right) \\
& \times H^{p} h^{p}\left(1+\left(1-2^{p}\right) \log _{2} \frac{H}{h}\right) \partial_{x}^{p+1} \partial_{y}^{p+1} c+\mathrm{O}\left(h^{p+1} \log _{2} \frac{1}{h}\right) .
\end{aligned}
$$

For third-order upwind discretization this yields

$$
\begin{aligned}
\widehat{d}= & -\frac{h^{3}}{12}\left(\int_{0}^{t}\left|a\left(t^{\prime}\right)\right| \mathrm{d} t^{\prime} \partial_{x}^{4}+\int_{0}^{t}\left|b\left(t^{\prime}\right)\right| \mathrm{d} t^{\prime} \partial_{y}^{4}\right) c \\
& +\frac{H^{p} h^{p}}{144}\left(1+\left(1-2^{p}\right) \log _{2} \frac{H}{h}\right) \sum_{n=0}^{M-1}\left(\int_{n t / M}^{(n+1) t / M}\left|a\left(t^{\prime}\right)\right| \mathrm{d} t^{\prime}\right)\left(\int_{n t / M}^{(n+1) t / M}\left|b\left(t^{\prime}\right)\right| \mathrm{d} t^{\prime}\right) \partial_{x}^{4} \partial_{y}^{4} c \\
& +\mathrm{O}\left(h^{p+1} \log _{2} \frac{1}{h}\right) .
\end{aligned}
$$

\section{Asymptotic efficiency}

When making efficiency comparisons the number of cell updates $C$ is used as a measure of required computational work. On a single grid this is simply defined as the product of the number of cells and the number of time steps required. Within the combination technique it is the sum of products of numbers of cells and time steps required on all grids within the grid of grids.

Due to the CFL restriction the time step $\Delta t$ must satisfy

$$
\Delta t \leqslant \alpha \min \left(\frac{h_{x}}{|a|}, \frac{h_{y}}{|b|}\right)
$$

for some constant value of $\alpha$. The cost estimates presented in this section are based on $\Delta t=$ $0.1 \min \left(h_{x}, h_{y}\right)$, as are the numerical results in Section 6. Note that the time steps on the different grids within the combination technique are not equal, larger steps are taken on coarser grids. In other words, within the combination technique the average CFL number (averaged over the semi-coarsened grids) is larger than the CFL number on the single grid. We identify a combination technique with a root mesh width $H=2 \cdot 2^{-L_{\mathrm{R}}}$, where $L_{\mathrm{R}}$ is the root level, and a finest mesh width $h=2 \cdot 2^{-L_{\mathrm{R}}-N}$, where $N$ is the sparseness level. The number of grids within a combination technique is given by

$$
2 N+1=2 \log _{2}\left(\frac{H}{h}\right)+1 .
$$

\subsection{Computational work}

Assuming the time interval $[0,1]$ and the spatial domain $[-1,1] \times[-1,1]$ for a single grid with $h=2 \cdot 2^{-L}$ the number of cell updates required is given by

$$
C_{1}=5 \cdot 2^{3 L} \text {. }
$$


For the combination technique the number of cell updates is given by

$$
\begin{aligned}
C_{\mathrm{CT}} & =\sum_{l+m=N-1, N} \frac{2^{l+m}}{\Delta t} \\
& =\sum_{l+m=N-1, N} \frac{2^{l+m}}{0.1 \cdot 2 \min \left(2^{-l}, 2^{-m}\right)} \\
& = \begin{cases}5 \cdot 2^{3 L_{\mathrm{R}}}\left(5 \cdot 2^{2 N}-4 \cdot 2^{3 N / 2}\right), & \text { for } N \text { even, } \\
5 \cdot 2^{3 L_{\mathrm{R}}}\left(5 \cdot 2^{2 N}-\frac{11}{4} \cdot 2^{(3 N+1) / 2}\right), & \text { for } N \text { odd. }\end{cases}
\end{aligned}
$$

For fixed $L_{\mathrm{R}}$ the combination technique has asymptotic complexity

$$
C_{\mathrm{CT}} \sim 2^{2 N} \sim h^{-2}
$$

while the single grid has asymptotic complexity

$$
C_{1} \sim 2^{3 L} \sim h^{-3} \text {. }
$$

\subsection{Efficiency comparison}

For fixed $L_{\mathrm{R}}$ the combination technique has, according to (25), the following asymptotic error:

$$
\widehat{d} \sim h^{p} \log _{2}\left(h^{-1}\right) \sim 2^{-p N} N
$$

while a single grid of mesh width $h=2 \cdot 2^{-L}$ has the following asymptotic error:

$$
d \sim h^{p} \sim 2^{-p L} .
$$

If we require a single grid to yield the same error as the combination technique for a given $N$, i.e., we put

$$
N 2^{-p N} \sim 2^{-p L},
$$

then we obtain

$$
L=N-\frac{\log _{2} N}{p} .
$$

According to (40) this yields, for the complexity of the single grid,

$$
C_{1} \sim 2^{3 N}\left(\frac{1}{N}\right)^{3 / p} \sim h_{\mathrm{CT}}^{-3}\left(\log _{2}\left(h_{\mathrm{CT}}^{-1}\right)\right)^{-3 / p},
$$

while according to (39), the complexity of the combination technique is given by

$$
C_{\mathrm{CT}} \sim 2^{2 N} \sim h_{\mathrm{CT}}^{-2}
$$

showing that, asymptotically, the combination technique reduces the three-dimensional single-grid complexity to a two-dimensional complexity, while obtaining the same level of accuracy.

\section{Numerical results}

\subsection{Numerical setup}

All the numerical results presented in this paper were obtained with the classical fourth-order explicit Runge-Kutta method using $\Delta t=0.1 \min \left(h_{x}, h_{y}\right)$ which satisfies the CFL condition for all considered 
test cases. We have verified that the time-discretization error is always negligible compared to the spatial discretization error. For spatial discretization we have used third-order upwind discretization as described in Section 2.2, the prolongations are done with fourth-order interpolation. All analytical error predictions for the combination technique refer solely to the combined discretization error. The interpolation and representation errors due to the combination technique are neglected. Boundaries are handled as in chapter 5 of [9], i.e., the exact solution is prescribed on the inlet boundaries and first order upwind discretization is used on the outflow boundaries. As a result boundary errors are introduced. These errors are not included in our analysis and were found to be insignificant for most of our numerical tests. See Section 6.4.1 for a further discussion of boundary errors.

\subsection{Test cases}

We consider the following four test cases:

(1) Horizontal advection, characterized by $a=\frac{1}{2}, b=0$.

(2) Diagonal advection with $a=b=\frac{1}{2}$.

(3) Time-dependent advection with

$$
(a, b)= \begin{cases}(0,2), & 0 \leqslant t<\frac{1}{4} \\ (2,0), & \frac{1}{4} \leqslant t<\frac{1}{2} \\ (0,-2), & \frac{1}{2} \leqslant t<\frac{3}{4} \\ (-2,0), & \frac{3}{4} \leqslant t<1\end{cases}
$$

(4) The Molenkamp-Crowley test case with $a=2 \pi y, b=-2 \pi x$.

Test cases (1)-(3) have as initial profile

$$
c(x, y, 0)=0.01^{4\left((x+0.25)^{2}+(y+0.25)^{2}\right)},
$$

which is depicted in Fig. 2(a), while test case (4) has as initial profile

$$
c(x, y, 0)=0.01^{4\left((x+0.5)^{2}+y^{2}\right)},
$$

which is depicted in Fig. 2(d). All test cases are integrated up to $t=1$ and have $-1 \leqslant x, y \leqslant 1$. In [9] solutions for the Molenkamp-Crowley test case obtained with various numerical methods are presented, given the initial condition (42). Our single grid solver is an implementation of the solver outlined in chapter 5 of [9]. Compared to the other solvers in [9] this solver is not the fastest but proved to be highly robust.

Besides initial profiles, Fig. 2 displays a number of typical error profiles observed in the numerical solutions of the test cases. The single-grid technique (SG) results in Fig. 2 were obtained on a $513 \times 513$ grid corresponding to $L=9$ and the combination technique (CT) used a grid of 9 grids given by $L_{r}=5$ and $N=4$, i.e., the combination technique also produced its solutions on a $513 \times 513$ grid. The results for the combination technique with intermediate combinations (ICT) were obtained by making 8 combinations.

Fig. 3 illustrates the performance of the single-grid and the combination technique on the test cases. The number of cell updates is plotted along the horizontal axis, which is a direct measure of the required CPU time, see Section 5.1. Any additional CPU time required to make the 7 intermediate combinations to obtain the ICT results was neglected, which is fully justified for the limited number of combinations considered here. The error is shown in the $L_{\infty}$ norm, the results for the $L_{1}$ norm are similar. In obtaining 


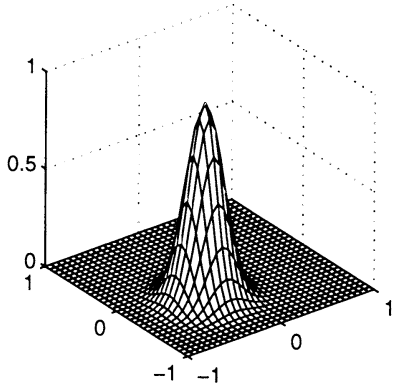

(a) Initial profile $1,2,3$

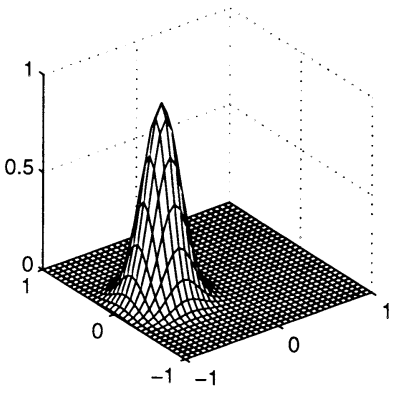

(d) initial profile 4

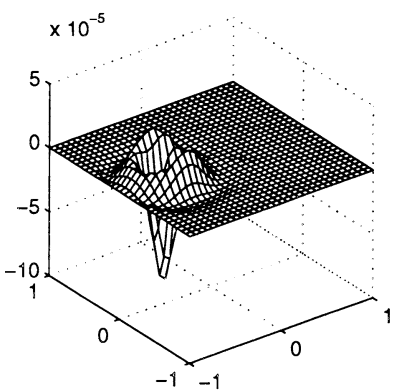

(g) Molenkamp, SG

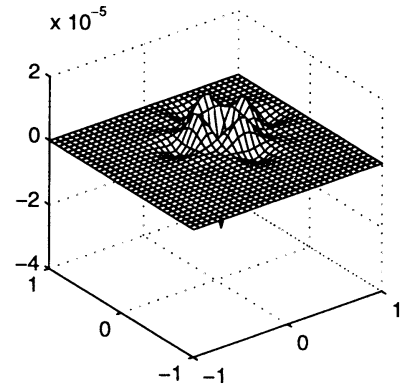

(b) diagonal, $\mathrm{CT}$

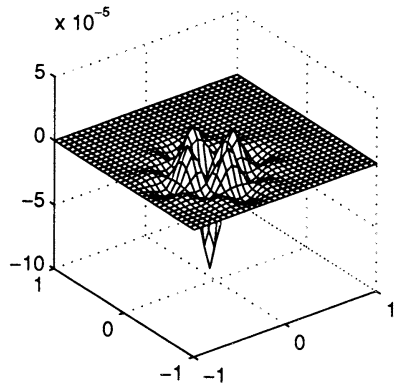

(e) time-dependent, CT

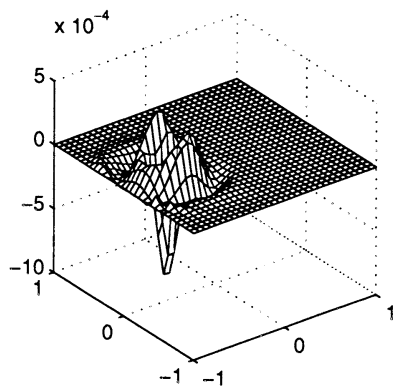

(h) Molenkamp, CT

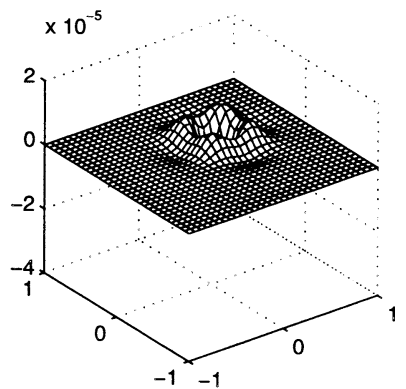

(c) diagonal, SG

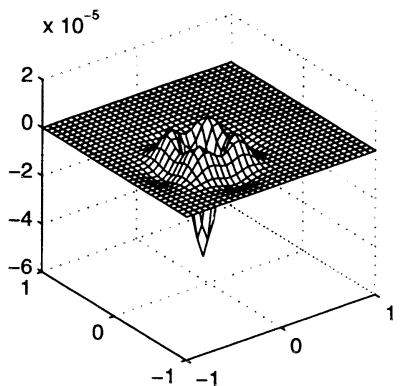

(f) time-dependent, ICT

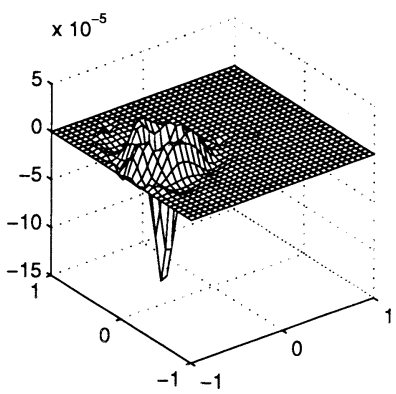

(i) Molenkamp, ICT

Fig. 2. Initial profiles and numerically observed errors for the single-grid technique (SG), the combination technique (CT) and the combination technique with intermediate combinations (ICT), applied to the diagonal, time-dependent and Molenkamp-Crowley test cases. 

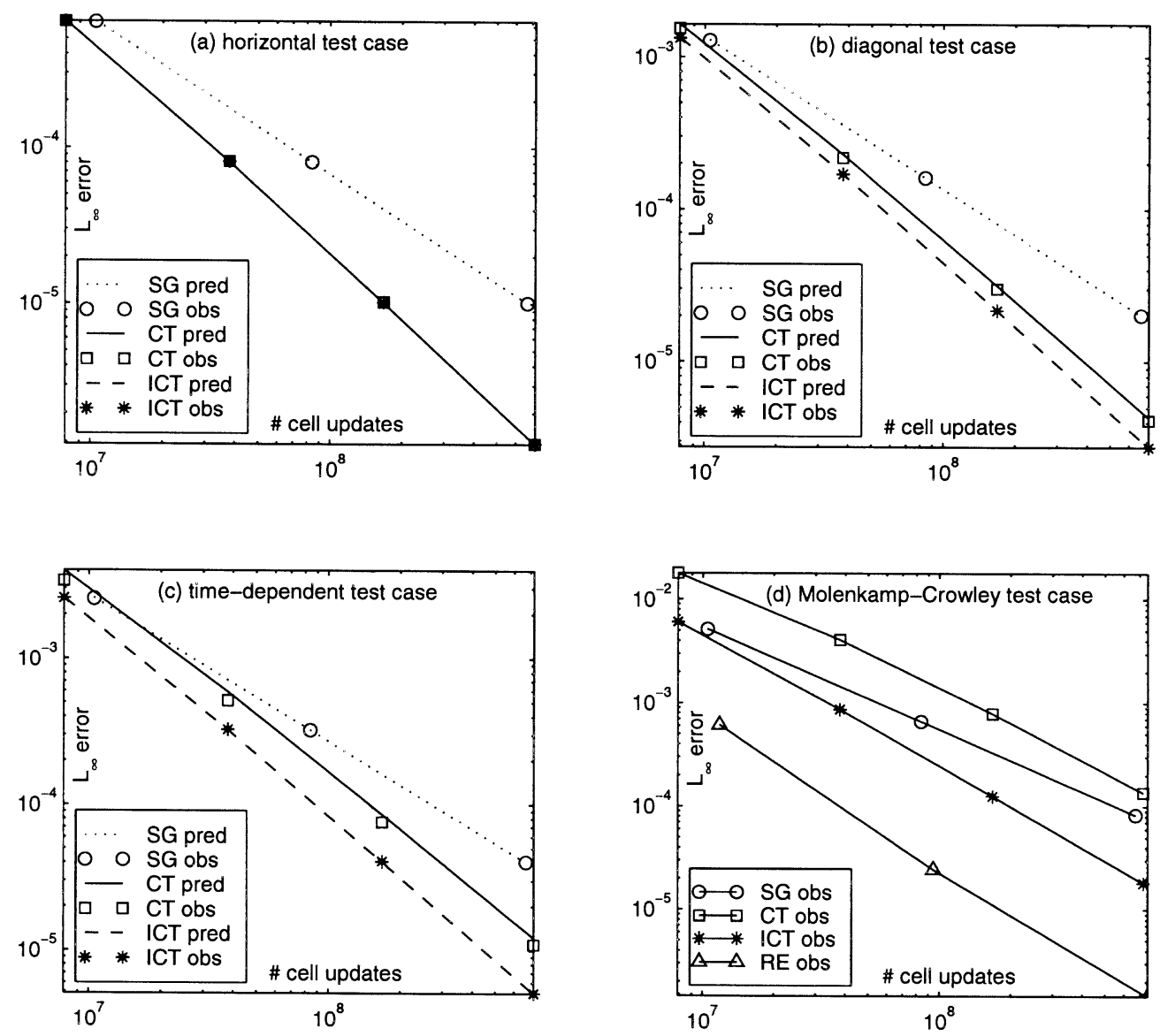

Fig. 3. Numerically observed (obs) and analytically predicted (pred) performance of the single-grid technique (SG), combination technique (CT), combination technique with intermediate combinations (ICT) and Richardson extrapolation (RE) applied to the test cases.

Fig. 3 the combination technique had $L_{r}=5$ fixed and $N=2,3,4,5$. The single-grid results were obtained using $L=7,8,9$.

In Fig. 4 the effect of the number of combinations is shown on the $L_{\infty}$ error due to a combination technique characterized by $L_{r}=5$ and $N=4$. In Fig. 4 only test cases (2)-(4) are considered because for test case (1) the error is independent of the number of combinations.

Except for numerically observed results, Figs. 3 and 4 also contain analytical predictions. For test cases (1) and (2) these were obtained from (10) for the single grid, from (26) for the combination technique and from (33) for the combination technique with intermediate combinations. For test case (3) the error predictions were obtained from (11) for the single grid, from (35) for the combination technique and from (37) for the combination technique with intermediate combinations. Note that test case (4) is not timedependent but spatially dependent. The error predictions that we have derived are not valid for spatially dependent coefficients. 

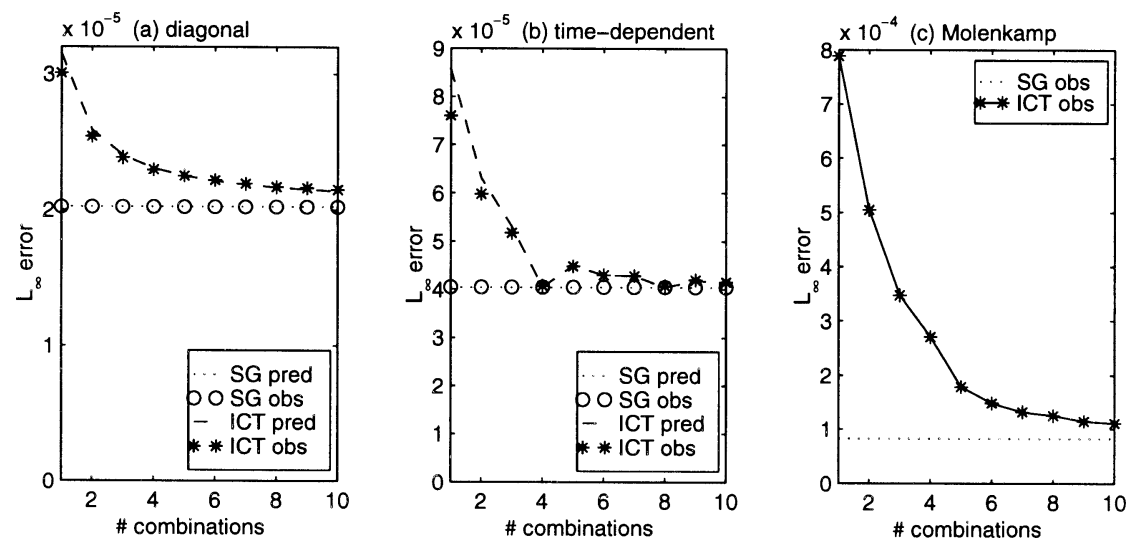

Fig. 4. $L_{\infty}$ error versus number of combinations for three test cases.

\subsection{Results}

\subsubsection{Horizontal test case}

We do not show any error profiles for the horizontal test case. For this test case the single-grid error and the errors due to the combination technique with and without intermediate combinations are all practically equal and are almost perfectly described by the analytical prediction (10). The combination technique does not introduce any additional error relative to the single grid because the second term in (26) vanishes due to $b=0$. The combination technique works very well for this fully grid-aligned test case, as can be seen in Fig. 3(a). Fig. 3(a) also shows that intermediate combinations do not improve the efficiency for the horizontal test case. In fact, the ICT results coincide with the CT results.

\subsubsection{Diagonal test case}

For the diagonal test case, error profiles are shown for the combination technique and the single grid in Figs. 2(b) and (c), respectively. We see that for this test case the error due to the combination technique is somewhat larger than the single grid error and has a different shape. Fig. 3(b) shows that the combination technique is more efficient than the single grid approach. This figure also shows that the combination technique can be made more efficient by applying 8 combinations. Fig. 4(a) shows how the error due to the combination technique decreases as the number of combinations is increased. The ICT error converges to the single-grid error as the number of combinations is increased. The first couple of combinations strongly decrease the error, a further increase in the number of combinations does not decrease the error much further.

\subsubsection{Time-dependent test case}

For the time-dependent test case the error profiles for the CT and the ICT are plotted in Figs. 2(e) and (f), respectively. We see that making intermediate combinations influences both the shape and size of the error. Note that Figs. 3(b) and (c) are similar, i.e., just like the diagonal test case the time-dependent test case is solved more efficiently with intermediate combinations (ICT) than without (CT). However, the reason for the efficiency of the ICT is somewhat more complex for the time-dependent test case than for the diagonal test case. As we can see from Fig. 4(b) the ICT error does not decrease monotonically 
with the number of combinations and this is correctly predicted by our theory. We can see that when a multiple of four combinations is made the ICT error becomes equal to the single grid error. This follows from (37) due to the fact that the product of integrals in the summation in the second term is always zero when a multiple of four combinations is made. When a multiple of four combinations is made the time-dependent test case is effectively split into two horizontal and two vertical advection problems and these are solved very well by the combination technique, as we know from the first test case.

For the time-dependent test case the agreement between predicted and observed error is very good for the single grid and the ICT. For the combination technique without intermediate combinations the agreement is a little weaker. This can be understood as follows. The combination technique tends to amplify cross-derivative terms in the single-grid error and of these amplified terms only one is included in our analytical predictions, viz. the second term in (26). The discrepancy between the predicted and observed CT errors is to be ascribed to the amplified cross-derivative terms that are not included in our analytical predictions. These terms are proportional to a second or higher power of $t$ and are therefore, according to Section 4.5, inversely proportional to a first or higher power of $M$ if $M$ combinations are made. Hence, the terms that cause the discrepancy are significantly smaller for the ICT than for the CT, especially for higher numbers of combinations.

\subsubsection{Molenkamp-Crowley test case}

Error profiles the Molenkamp-Crowley test case are shown in Figs. 2(g), (h) and (i) for the SG, CT and ICT, respectively. We see that the CT error is larger than the SG error, but intermediate combinations help considerably, i.e., the ICT error lies much closer to the SG error than to the CT error. Fig. 3(d) shows that the Molenkamp-Crowley test case is a tough case to solve efficiently with the combination technique. Fig. 3(d) shows that CT is less efficient than the single-grid technique, whereas ICT is more efficient in solving the Molenkamp-Crowley test case. For completeness, Fig. 4(c) shows how the ICT error decreases with increasing number of combinations. It is interesting to note that the ICT performs so much better than the CT for the Molenkamp-Crowley test case. This is not really surprising since this test case is clearly not well suited to any semi-coarsened grid because it models advection in all directions. Therefore the CT solution, which is constructed from solutions on semi-coarsened grids, is not very accurate either. By allowing sufficient intermediate combinations the test case is split into problems that do have a dominant direction of advection and therefore are more suited to some semi-coarsened grid. The corresponding ICT solution is also more accurate.

\subsection{Implementational issues}

\subsubsection{Boundary complications}

The $L_{\infty}$ errors for the Molenkamp-Crowley test case were determined after the solutions were restricted to the $33 \times 33$ root grid. We were forced to do this because at high accuracies the fourthorder interpolation produced wiggles near the boundaries that dominate the combined discretization error. These wiggles do not appear in the nodes of the root grid, because for those nodes no interpolation is necessary. However, at very high resolution wiggles near the boundaries appear in the nodes of the root grid as well. In particular, for $L_{\mathrm{R}} \geqslant 6$ the wiggles are of equal or greater magnitude than the combined discretization error itself. The cause for these wiggles lies in the fact that the discretization near the boundaries is of lower order which obstructs the cancellation of errors required by the combination technique to function properly. An illustration of wiggles near the boundary is shown in Fig. 5(b). The 


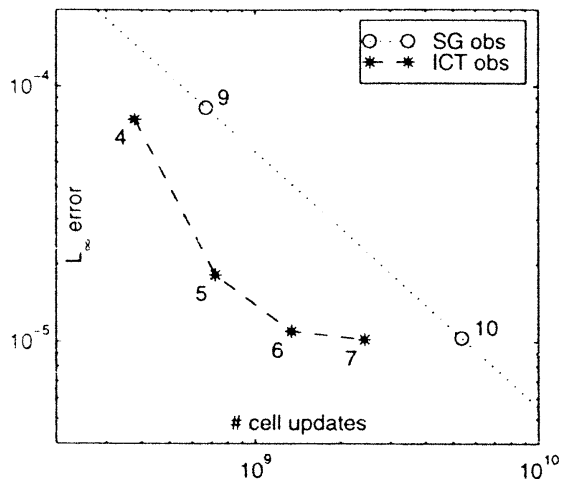

(a)

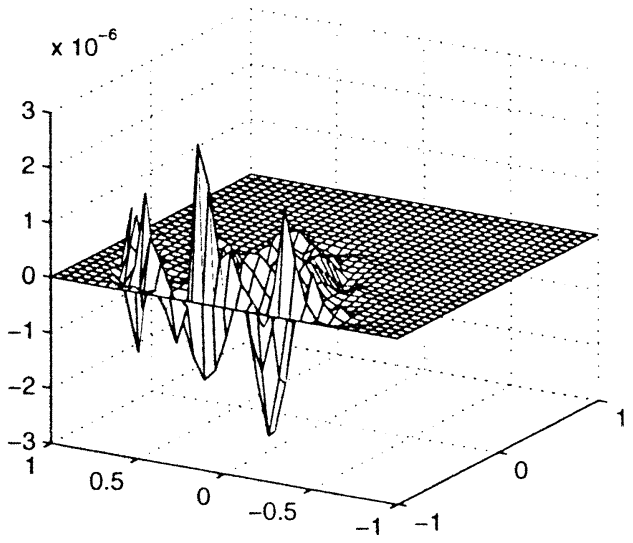

(b)

Fig. 5. Implementational issues; Molenkamp-Crowley test case. (a) Performance of the combination technique with 8 combinations (ICT) for root levels 4-7. (b) Error profile due to a combination technique with root level 5, sparseness level 6 and 8 combinations.

above difficulties were not observed for the other test cases because there the solutions are almost zero near the boundaries. We also ran the Molenkamp-Crowley test case for the initial profile (41) shown in Fig. 2(a) which stays away from the boundaries. This removed the problems near the boundaries but introduced a similar wiggle in the origin. We believe that this wiggle is also due to an order reduction caused by the switching of the upwind discretization stencil in horizontal and vertical directions due to the sign change of the coefficients in the origin.

\subsubsection{Choosing an optimal root mesh-width}

All numerical results for the combination technique were obtained with a root mesh width $H=\frac{1}{16}$ corresponding to a root level $L_{\mathrm{R}}=5$. This choice was made to optimize the performance of the combination technique when applied to the Molenkamp-Crowley test case. This is illustrated in Fig. 5(a). In this figure the performance of the combination technique with 8 combinations which has $L_{\mathrm{R}}+N=10$ fixed (ICT) is compared with the single-grid performance (SG). We see that for $L_{\mathrm{R}}=5$ the performance of the ICT is optimal, although performance for $L_{\mathrm{R}}=6$ is comparable. The optimal choice for $L_{\mathrm{R}}$ is only weakly dependent on the sparseness level $N$, therefore we could safely use $L_{\mathrm{R}}=5$ throughout for optimal performance. To see that the optimal $L_{\mathrm{R}}$ varies slowly with $N$ consider the following argument. We found that, to solve the Molenkamp-Crowley test efficiently, the additional error due to the combination technique had to be of comparable magnitude as the single-grid error. According to our error analysis for constant coefficients (26) this implies

$$
h^{3} \sim H^{3} h^{3} \log _{2} \frac{H}{h}
$$

which leads to

$$
H \sim\left(\frac{1}{N}\right)^{1 / 3},
$$




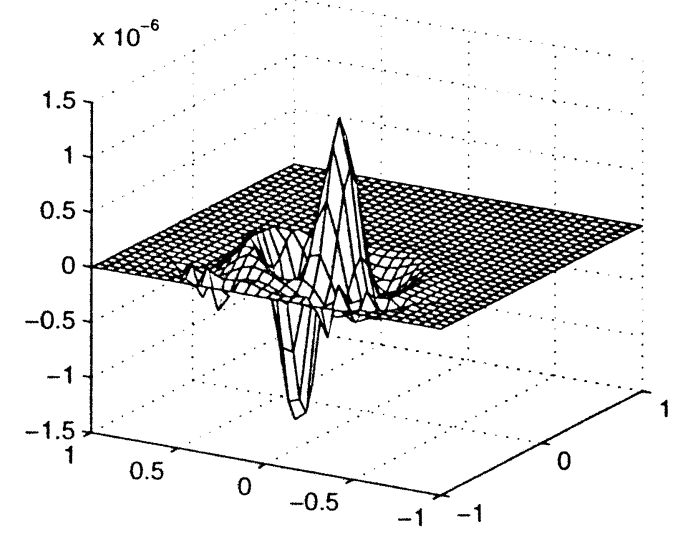

Fig. 6. Error profile present in an $N=9$ Richardson extrapolant.

showing that $H$ needs to decrease only slightly when the sparseness level, and thus the number of grids in the combination technique, increases.

\subsection{Richardson extrapolation}

In [7] Rüde points out that simple Richardson extrapolation is in fact more efficient than the combination technique for the solution of a smooth Poisson problem. To see how Richardson extrapolation would perform for the Molenkamp-Crowley test case, we considered the following Richardson extrapolant

$$
\omega_{\mathrm{R}}^{N, N} \equiv \frac{8}{7} \omega^{N, N}-\frac{1}{7} P^{N, N} \omega^{N-1, N-1},
$$

which cancels the leading third-order term in the error expansion (10). The new leading-order terms are proportional to $h^{4} \partial_{x}^{5} c$ and $h^{4} \partial_{y}^{5} c$ and are thus of a dispersive nature which is shown in the $N=9$ error profile for Richardson extrapolation in Fig. 6. The Richardson extrapolant has an asymptotic error

$$
d_{\mathrm{RE}} \sim h_{\mathrm{RE}}^{4}
$$

while it has the same asymptotic complexity as a single grid,

$$
C_{\mathrm{RE}} \sim h_{\mathrm{RE}}^{-3} \text {. }
$$

If we consider a combination technique and a Richardson extrapolation of equal complexity, i.e., we put

$$
C_{\mathrm{RE}} \sim C_{\mathrm{CT}}
$$

then we obtain

$$
h_{\mathrm{RE}} \sim h_{\mathrm{CT}}^{2 / 3}
$$

which leads to

$$
d_{\mathrm{RE}} \sim h_{\mathrm{CT}}^{8 / 3} \text {. }
$$


According to (26) the combination technique has

$$
\widehat{d} \sim h_{\mathrm{CT}}^{3} \log h_{\mathrm{CT}}^{-1} \text {. }
$$

Comparison of (43) with (44) shows that in the limit $h \rightarrow 0$ the combination technique shall be more efficient than Richardson extrapolation.

In Fig. 3(d) the numerically observed performance of Richardson extrapolation (RE) is compared with that of the single grid (SG) and the combination technique with intermediate combinations (ICT) when applied to the Molenkamp-Crowley test case. Fig. 3(d) clearly shows that Richardson extrapolation is very efficient for the Molenkamp-Crowley test case, much more so than the combination technique, even though we expect the combination technique to be superior to Richardson extrapolation in the asymptotic limit $h \rightarrow 0$. For the Molenkamp-Crowley test case, without parallelization and on grids of practically relevant mesh width, the combination technique can not compete with Richardson extrapolation. Note that Richardson extrapolation and the combination technique strive for higher efficiency in different ways. Richardson extrapolation generates a higher-order solution for a marginally larger complexity, while the combination technique requires lower complexity for a marginally larger error.

\section{Conclusions}

We have derived leading-order expressions for the error that is introduced when a spatially constant coefficient advection equation is solved with the combination technique. In our derivations we have accounted for time-dependent coefficients and for intermediate combinations. When a constant coefficient advection equation

$$
c_{t}+a c_{x}+b c_{y}=0
$$

is solved on a grid of mesh width $h$, this will introduce an error $d$ into the numerical solution which is in leading order given by

$$
d=t \phi h^{p}\left(|a| \partial_{x}^{p+1}+|b| \partial_{y}^{p+1}\right) c+\mathrm{O}\left(h^{p+1}\right)
$$

where $c$ is the exact solution, $p$ is the order of discretization and $\phi$ is an error constant. We have shown that when we solve (45) with the combination technique, we obtain an error $\widehat{d}$ which is in leading order given by

$$
\begin{aligned}
\widehat{d}= & t \phi h^{p}\left(|a| \partial_{x}^{p+1}+|b| \partial_{y}^{p+1}\right) c+\frac{1}{M} t^{2} \phi^{2}|a b| H^{p} h^{p}\left(1+\left(1-2^{p}\right) \log _{2} \frac{H}{h}\right) \partial_{x}^{p+1} \partial_{y}^{p+1} \\
& +\mathrm{O}\left(h^{p+1} \log _{2} \frac{1}{h}\right),
\end{aligned}
$$

where $H$ is the mesh width of the coarsest grid in the combination technique and $M$ is the number of combinations. We see that the leading-order term from the single grid error (46) reappears in the combination technique error (47) and is accompanied by a new term which is formally of order $h^{p} \log h^{-1}$. Focusing only on the order in terms of $h$, this new term has to be identified as the leadingorder term in (47). The numerical experiments suggest, however, that the term proportional to $h^{p}$ in (47), which is also present in the single-grid error, is of equal importance as the new term proportional to $h^{p} \log h^{-1}$. The additional error due to the combination technique, corresponding to the second term 
in (47), is proportional to $1 / M$. This suggests that the error due to the combination technique can be strongly reduced by making several of intermediate combinations. The numerical results confirm this. For our test case that has time-dependent coefficients it turns out that the number of combinations has to be chosen such that the problem is split up in problems which have a constant direction of advection. This agrees with our error analysis. Finally, the combination technique proved more efficient for gridaligned problems than for non-grid-aligned problems, which follows from numerical observations and from analysis.

For the Molenkamp-Crowley test simple Richardson extrapolation proved more efficient than the combination technique, even though the combination technique is expected to be more efficient in the asymptotic limit $h \rightarrow 0$. Rüde made the same observation for a smooth Poisson problem in [7].

When going to three spatial dimensions (or even higher dimensional problems), the combination technique will perform significantly better. Furthermore, very significant gains in performance can be obtained when the combination technique is parallelized.

\section{Appendix. Notation}

$\Omega^{N, N} \quad$ finest grid of mesh width $h=2^{-N} H$.

$\Omega^{l, m} \quad$ semi-coarsened grid of mesh widths $h_{x}=2^{-l} H, h_{y}=2^{-m} H$.

$\Omega^{0,0} \quad$ root grid of mesh width $h=H$.

$c \quad$ continuous, exact solution.

$R^{l, m} \quad$ restriction operator that maps onto $\Omega^{l, m}$.

$P^{N, N} \quad$ prolongation operator that maps onto $\Omega^{N, N}$.

$\omega^{l, m} \quad$ semi-discrete approximate solution on $\Omega^{l, m}$.

$h \quad$ mesh width of grid $\Omega^{N, N}$.

$H \quad$ mesh width of root grid $\Omega^{0,0}$.

$d \quad$ discretization error: $d \equiv \omega-c$.

$E \quad$ truncation error operator: $E \equiv a D_{x}+b D_{y}+\mathrm{d} / \mathrm{d} t$.

$D_{x}, D_{y}$ finite difference approximations of $\partial_{x}, \partial_{y}$.

$a, b \quad$ advection speeds in $x$ and $y$ direction.

$x, y \quad$ spatial coordinates.

$t \quad$ time coordinate.

$S_{h_{x}} \quad x$ shift operator: $S_{h_{x}} f(x, y) \equiv f\left(x+h_{x}, y\right)$.

$S_{h_{y}} \quad y$ shift operator: $S_{h_{y}} f(x, y) \equiv f\left(x, y+h_{y}\right)$.

$l, m \quad$ grid indices: $0 \leqslant l, m \leqslant N$. 
$\widehat{c}^{N, N} \quad$ sparse grid approximation of exact solution:

$$
\widehat{c}^{N, N} \equiv \sum_{l+m=N} R^{l, m} c-\sum_{l+m=N-1} R^{l, m} c .
$$

$\widehat{\omega}^{N, N} \quad$ sparse grid combination of numerical solutions:

$$
\widehat{\omega}^{N, N} \equiv \sum_{l+m=N} \omega^{l, m}-\sum_{l+m=N-1} \omega^{l, m} .
$$

$r^{N, N} \quad$ representation error: $r^{N, N} \equiv \widehat{c}^{N, N}-R^{N, N} c$.

$e^{N, N} \quad$ total error: $e^{N, N} \equiv \widehat{\omega}^{N, N}-R^{N, N} c$.

$\theta_{i, j} \quad$ expansion coefficients of the discretization error:

$$
d^{l, m}=\sum_{i=0}^{\infty} \sum_{j=0}^{\infty} h_{x}^{i} h_{y}^{j} R^{l, m} \theta_{i, j} c .
$$

$\varepsilon_{i, j}^{l, m} \quad$ error term: $\varepsilon_{i, j}^{l, m} \equiv 2^{-i l-j m} R^{l, m} \theta_{i, j} c$.

$\xi_{i, j}^{N, N, l, m}$ error without interpolation effects: $\xi_{i, j}^{N, N, l, m} \equiv 2^{-i l-j m} R^{N, N} \theta_{i, j} c$.

$\zeta_{i, j}^{N, N, l, m}$ additional error due to interpolation: $\zeta_{i, j}^{N, N, l, m} \equiv\left(\lambda_{t} h_{x}^{q} \partial_{x}^{q}+\lambda_{m} h_{y}^{q} \partial_{y}^{q}\right) \xi_{i, j}^{N, N, l, m}$.

$\lambda_{l}, \lambda_{m} \quad$ constants dependent on the choice of interpolation.

$\alpha_{p}, \beta_{p} \quad$ constants dependent on the choice of interpolation.

$p \quad$ order of spatial discretization.

$q \quad$ order of interpolation used in prolongation.

$N \quad$ sparseness level of combination technique.

$M \quad$ total number of combinations.

$C_{1} \quad$ number of cell updates on a single grid.

$C_{\mathrm{CT}} \quad$ number of cell updates within the combination technique.

$\omega_{\mathrm{R}}^{N, N} \quad$ Richardson extrapolant: $\omega_{\mathrm{R}}^{N, N} \equiv \frac{8}{7} \omega^{N, N}-\frac{1}{7} \omega^{N-1, N-1}$.

$d_{\mathrm{RE}} \quad$ error in Richardson extrapolant: $d_{\mathrm{RE}} \equiv \omega_{\mathrm{R}}^{N, N}-R^{N, N} c$.

$C_{\mathrm{RE}} \quad$ number of cell updates required for Richardson extrapolation.

\section{References}

[1] H.J. Bungartz, M. Griebel, D. Roschke, C. Zenger, Pointwise convergence of the combination technique for the Laplace equation, East-West J. Numer. Math. 2 (1994) 21-45.

[2] C.T.H. Everaars, B. Koren, Using coordination to parallelize sparse-grid methods for 3D CFD problems, Parallel Comput. 24 (1998) 1081-1106. 
[3] M. Griebel, The combination technique for the sparse grid solution of PDE's on multiprocessor machines, Parallel Process. Lett. 2 (1992) 61-70.

[4] M. Griebel, M. Schneider, C. Zenger, A combination technique for the solution of sparse grid problems, in: R. Beauwens, P. de Groen (Eds.), Iterative Methods in Linear Algebra, North-Holland, Amsterdam, 1992, pp. 263-281.

[5] M. Griebel, G. Zumbusch, Adaptive sparse grids for hyperbolic conservation laws, in: M. Fey, R. Jeltsch (Eds.), Hyperbolic Problems: Theory, Numerics, Applications, International Series of Numerical Mathematics, Vol. 129, Birkhäuser, Basel, 1999, pp. 411-422.

[6] B. Lastdrager, B. Koren, Error analysis for function representation by the sparse-grid combination technique, Report MAS-R9823, CWI, Amsterdam, 1998.

[7] U. Rüde, Multilevel, extrapolation and sparse grid methods, in: P.W. Hemker, P. Wesseling (Eds.), Multigrid Methods IV, International Series of Numerical Mathematics, Vol. 116, Birkhäuser, Basel, 1993, pp. 281-294.

[8] J.G. Verwer, W.H. Hundsdorfer, J.G. Blom, Numerical time integration for air pollution models, Report MASR9825, CWI, Amsterdam, 1998.

[9] C.B. Vreugdenhil, B. Koren (Eds.), Numerical Methods for Advection-Diffusion Problems, Notes on Numerical Fluid Mechanics, Vol. 45, Vieweg, Braunschweig, 1993.

[10] C. Zenger, Sparse grids, in: W. Hackbusch (Ed.), Notes on Numerical Fluid Mechanics, Vol. 31, Vieweg, Braunschweig, 1991, pp. 241-251. 